\title{
Role of curcumin in idiopathic pulmonary arterial hypertension treatment: A new therapeutic possibility is
}

\author{
Enrico Bronte ${ }^{\mathrm{a}, *}$, Giuseppe Coppola ${ }^{\mathrm{b}}$, Riccardo Di Miceli ${ }^{\mathrm{c}}$, Vincenzo Sucato ${ }^{\mathrm{b}}$, Antonio Russo $^{\mathrm{a}}$, \\ Salvatore Novo ${ }^{\text {b }}$ \\ a Department of Surgical, Oncological and Stomatological Sciences, University of Palermo, Palermo, Italy \\ ${ }^{\mathrm{b}}$ Department of Internal Medicine and Cardiovascular Disease, University of Palermo, Palermo, Italy \\ ${ }^{\mathrm{c}}$ Department of Internal Medicine, University of Palermo, Palermo, Italy
}

\section{A R T I C L E I N F O}

\section{Article history:}

Received 31 January 2013

Accepted 11 August 2013

\begin{abstract}
A B S T R A C T
The idiopathic pulmonary arterial hypertension is a complex disease that mainly affects pulmonary arterial circulation. This undergoes a remodeling with subsequent reduction of flow in the small pulmonary arteries. Because of this damage an increased vascular resistance gradually develops, and over time it carries out in heart failure. The inflammatory process is a key element in this condition, mediated by various cytokines. The inflammatory signal induces activation of NF- $\kappa \mathrm{B}$, and prompts TGF- $\beta$-related signaling pathway. Clinical evolution leads to progressive debilitation, greatly affecting the patient quality of life. The actual therapeutic approaches, are few and expensive, and include systemic drugs such as prostanoids, phosphodiesterase inhibitors and antagonists of endothelin-1 (ERBs). Some researchers have long investigated the anti-inflammatory effects of curcumin. It shows a role for inactivation of NF-KB-mediated inflammation. On the basis of these findings we propose a potential role of curcumin and its pharmacologically fit derivatives for treatment of idiopathic pulmonary arterial hypertension.
\end{abstract}

(c) 2013 Elsevier Ltd. All rights reserved.

\section{Introduction}

The pulmonary arterial hypertension was considered for a long time a rare disease. In 2006 from a French registry its prevalence has been estimated in about 15 per million. The idiopathic pulmonary arterial hypertension (IPAH) is more prevalent in women and is the most common type of pulmonary arterial hypertension. An arterial remodeling, affecting the arteries of the pulmonary arterial circulation, underlies this complex disorder. It leads to a reduction of flow in the small arteries, which develops increased vascular resistance, and over time it ends in heart failure [1]. This condition is the result of a sum of events, as it is the case for diseases such as atherosclerosis or systemic arterial hypertension. Among these events some researchers identified BMPR2 gene germline mutation, which codes for a protein belonging to TGF $\beta$ family. BMPR2 modulates the vascular cells growth via activation of SMAD and LIM kinases. Therefore the mutation leads to an alteration of signal transduction within the vascular smooth muscle cells with alteration of the balance between cell proliferation and apoptosis [2-4]. From an histo-pathological point of view, trigger-

\footnotetext{
The authors did not receive grants for this work.

* Corresponding author. Address: Department of Surgical, Oncological and Stomatological Sciences, University of Palermo, Via del Vespro n. 129, 90127 Palermo, Italy. Tel.: +39 0916552500

E-mail address: enrico.bronte@gmail.com (E. Bronte).
}

ing events are intimal hyperplasia, medial hypertrophy, adventitial proliferation with in situ thrombosis, various degrees of inflammation and plexiform arteriopathy. Thus, the inflammatory process is clearly a key element in the disease, as a matter of fact the B and T lymphocytes, dendritic cells and macrophages involved, are all called due to an increase in serum levels of cytokines IL-1 $\beta$, IL-6, IL-8 or TNF $\alpha$. Chemokines (CCL-2/MCP-1, CCL-5/RANTES) also contribute to it $[2,3,5]$. So it is explained why the pulmonary arterial hypertension is often associated with "inflammatory" conditions as polyneuropathy syndrome, systemic lupus erythematosus, scleroderma, Hashimoto's thyroiditis $[1,6]$. Growth factors contribute to the inflammatory process, together with cytokines, affecting the remodeling that develops during the course of this disease. They are PDGF (platelet-derived growth factor) that is expressed in plexiform lesions together with the VEGF receptor 2 (VEGFR2), EGF (epidermal growth factor), VEGF (vascular endothelial growth factor), FGF2 (fibroblast growth factor 2). Altered levels of bFGF (basic) were detected in plasma and urine of patients with pulmonary arterial hypertension. The inflammatory process involves various ways that have as protagonists several transcription factors. It is important among these, the signaling activity of NF- $\kappa B$ (nuclear factor $\kappa \mathrm{B}$ ), which is activated by several cytokines and extracellular inflammatory triggers. The other major route, as already seen, is the signaling pathway that involves the factors belonging to the TGF $\beta$-signaling family $[2,3]$. The IPAH most common symptom is dyspnea on exertion. It indicates a progressive 
lowering in oxygen transport, cardiac output, diffusing capacity of carbon monoxide and mixed venous oxygen saturation, leading to hypoxemia and increased work of breathing. Other symptoms are fatigue, lack of energy, angina, syncope, palpitations and peripheral edema. Edema results from right ventricular failure and tricuspid regurgitation. The physical examination shows accentuated pulmonary component of S2, pansystolic murmur of tricuspid regurgitation, jugular vein distension, hepatomegaly and ascites $[5,8]$.

\section{Therapeutic approaches}

They can be distinguished in a basal treatment and a specific therapy. The first one includes diuretics, mainly loop diuretics and spironolactones, for right heart failure treatment and other drugs for left heart failure such as angiotensin converting enzyme inhibitors or angiotensin II receptor blockers or digoxin, chronic oxygen and anticoagulants. On the other hand, there is the specific medical therapy for idiopathic pulmonary arterial hypertension. For a long time only calcium channel blockers were used, but it was clarified that only a little percent of patients responds to them. So the current specific therapy took over. There are mainly three types of drug, by acting on different routes, alone or in association. Prostanoids, prostacyclin analogs, restore the imbalance between thromboxane A2, a vasoconstrictor molecule, and prostacyclin, a vasodilator molecule. This imbalance in favor of thromboxane A2 produces vasoconstriction to which thrombosis and platelet activation contribute. Phosphodiesterase inhibitors inhibiting PDE5 (Phosphodiesterase 5) prolong the time of permanence within the cell of cGMP. This molecule belongs to the signaling pathway starting from NO, thus producing a vasodilator effect for relaxation of vascular smooth muscle cells. Another class of drugs in use is represented by antagonists of endothelin-1 (ERBs). The latter is a potent endogenous vasoconstrictor molecule, which is mitogen on smooth muscle cells and therefore it also contributes to pulmonary vascular hypertrophy [7,8]. The limit of the actual treatments is that they are few, expensive and even more in a combination therapy, and they have various side effects. The most common are pain, headache, nausea, diarrhea and in some cases liver toxicity.

\section{Curcumin as a possible therapeutic agent from diet}

Turmeric (prepared from the root of the Curcuma longa) belongs to the ginger family. It is more commonly available as a dried powder, curry powder. Curry is a spice used for decades in traditional Indian medicine for the treatment of various diseases such as arthritis, ulcers, wounds, fever, trauma, as well as skin diseases, for example psoriasis. Main active constituent of turmeric is curcumin, whose active constituent is diferuloylmethane, a hydrophobic polyphenol, which gives the characteristic yellow-orange color to curry $[9,10]$. Curcumin, demethoxycurcumin, bisdemethoxycurcumin and the more recent cyclocurcumin are the major components (called curcuminoids) isolated from turmeric over the years. Over the past 60 years studies have proven antioxidant, antibacterial, antifungal, antiviral, anti-inflammatory, anti-proliferative, proapoptotic and anti-atherosclerotic properties. These components exert beneficial effects to various diseases including cardiovascular disease and pulmonary fibrosis. The molecular targets are numerous, the curcumin can bind them directly or modulate their activities or indirectly regulate the function. It has been seen that more than 30 proteins interact directly with curcumin, including DNA polymerase, protein kinase $C$, lipoxygenase. Growth factors, transcription factors, kinases, inflammatory cytokines, adhesion molecules, apoptosis-related proteins are its molecular targets. As regards the transcription factors curcumin potently inhibits the activation of NF- $\kappa$ B (nuclear factor $\kappa \mathrm{B}$ ), STAT proteins, HIF-1 (hypoxia-inducible factor-1), while on the other hand activates PPAR$\gamma$ (peroxisome proliferator activated receptor- $\gamma$ ). The nuclear factors NF- $\kappa$ B, STAT-3, HIF-1 are involved in cell proliferation, cell survival, invasion, angiogenesis and inflammation [9]. It has been shown that curcumin reversibly inhibits STAT3 activation in some cells and by this mechanism it suppresses IL-6-induced cell proliferation [11]. In inflammatory diseases there is a dysregulation of $\mathrm{NF}-\kappa \mathrm{B}$. Normally in unstimulated cells, NF- $\kappa \mathrm{B}$ is constitutively localized in the cytosol as a heterodimer bound to an inhibitory protein called $\mathrm{I} \kappa \mathrm{B}$, various pathogenic stimuli including cytokines, ischemia/reperfusion can activate NF- $\kappa B$ through various pathways. Under activation NF- $\kappa B$ is translocated to the nucleus, where it induces the expression of several target genes, that play a role in inducing cell proliferation, invasion, inflammation. Curcumin prevents NF- $\kappa B$ activation induced by various agents through the inhibition of translocation of $\mathrm{p} 65$ to the nucleus and suppressing $\mathrm{I} \kappa \mathrm{B} \alpha$ degradation in numerous cytotypes. Curcumin, by inhibiting NF$\kappa \mathrm{B}$ activation, suppresses the expression of various cell survival and proliferative genes, including IL6 and cyclooxygenase 2, it inhibits proliferation and induces apoptosis. Curcumin modulates the expression and activity of some growth factors, including TGF $\alpha$ (transforming growth factor $\alpha$ ), EGFR (epidermal growth factor receptor), a member of the ErbB family receptors. In this case, curcumin blocks the signaling pathway EGFR preventing tyrosine phosphorylation and suppressing the EGFR gene expression, that is mediated by activation of PPAR- $\gamma$ [9]. It may directly inhibit angiogenesis and also downregulate expression of vascular endothelial growth factor (VEGF), fibroblast growth factor (FGF) and EGF [12]. Interleukins are another group of inflammatory cytokines that play a critical role in the regulation of inflammation response, as well as signaling pathways such as NF- $\kappa B$ and the STATs [9]. Inhibition of pro-inflammatory cytokine production by regulation of transcriptional factors, such as NF- $\kappa B$, is a potential strategy for controlling inflammatory responses $[13,14]$. Excessive synthesis and production of pro-inflammatory cytokines, including TNF$\alpha$, IL-1 $\beta$ and IL-6, play a major role in the development of local and systemic inflammation, causing severe pathophysiological derangement or organ failure [15]. Several studies have demonstrated that curcumin was able to modulate the production of various inflammatory cytokines, thereby exhibiting potent antiinflammatory activity $[16,17]$. It reduces the expression of IL-1 $\beta$, IL-6 and IL- 8 through suppression of NF- $\kappa B$ and MAPK pathways $[9,10]$. TNF- $\alpha$ plays an important role in immune cells regulation and the development of systemic inflammation. Dysregulation of TNF- $\alpha$ production has been implicated in a variety of inflammatory diseases. Curcumin has profound inhibitory effects on the production of TNF- $\alpha$ and it also showed effects on modulation of TNF- $\alpha$ induced signaling [9]. It can down-regulate or suppress NF- $\kappa \mathrm{B}$ activation induced by TNF- $\alpha$. The mechanism appears to be via a reduced IkBa phosphorylation and degradation, suggesting that curcumin acts at a step above IkB kinase (IKK) in the NF- $\kappa B$ activation pathway. Many of the observed biological effects of curcumin involve processes that are NF- $\kappa B-d e p e n d e n t ~[10]$. In endothelial cells, treatment with curcumin suppressed TNF-induced NF- $\kappa B$ activation and the expression of adhesion molecules, thereby inhibiting the adhesion of monocytes to endothelial cells [18]. Enzymes closely associated with inflammation like COX-2, inducible nitric oxide synthase (iNOS), 5-LOX, and phospholipases A2 (PLA2) are modulated by curcumin. It can downregulate the expression and the activity of COX-2 both in vitro and in vivo [9]. Curcumin inhibits COX2 production and inhibits COX2 induction. The COX2 gene promoter contains two NF- $\kappa \mathrm{B}$ binding sites and show evidence that the effect of curcumin on COX2 is due to inhibition of NF- $\kappa B$ binding. The level of impact of curcumin upon the NF- $\kappa \mathrm{B}$ pathway appears to be at or above IKK. A very important 
aspect is that inhibition of COX2 by curcumin lacks the adverse effects of chronic aspirin or non-steroidal anti-inflammatory drugs ingestion [10]. Inhibition of NF- $\kappa B$ completely blocked TNF- $\alpha$-induced expression of ICAM-1, VCAM-1, and E-selectin, indicating that the expression of CAMs is regulated in part by NF- $\kappa \mathrm{B}$. Curcumin potently inhibited TNF- $\alpha$-induced expression of ICAM-1, VCAM-1, and ELAM-1 partially through NF- $\kappa B$ inhibition. As regards the pharmacokinetics, safety and efficacy of curcumin, it is remarkably well tolerated. Even at high doses, curcumin appears nontoxic to humans. If curcumin is administered as a single daily oral dose ranging from 500 to $8000 \mathrm{mg} /$ day for 3 months, the treatment is well tolerated [9]. Oral doses up to $12 \mathrm{~g} /$ day are well tolerated in human subjects [19], although dosing diet regimen above $8 \mathrm{~g}$ may be difficult to achieve due to the bulky nature of this quantity of compound [20]. Although curcumin is well tolerated and has a wide variety of beneficial activities and possesses good pharmacological properties, its bioavailability in vivo is poor. This may be an important obstacle to its utility as a potential therapeutic agent [9]. Drug delivery is a problem and the bioavailability of oral curcumin is low due to a combination of rapid and efficient first pass metabolism, poor gastrointestinal absorption, rapid systemic elimination and poor aqueous solubility [10,21]. A clinical study showed that serum levels of curcumin were undetectable or very low after $2 \mathrm{~g}$ of pure curcumin powder was administered alone to fasting volunteers [22]. Curcumin undergoes avid glucuronidation and sulfation leading to the formation of curcumin glucuronide and, less commonly, curcumin sulfates. It was suggested that the low systematic bioavailability of curcumin may be due to the hydrophobic nature of the molecule, poor absorption and the metabolic biotransformation in intestine and liver [23]. These conjugates are detectable in plasma at greater concentrations than free curcumin with a peak at $4 \mathrm{~h}$ after oral dosing [24]. This may be explained by the fact that curcumin constitutes about $5 \%$ of turmeric root; the remainder is made up of carbohydrates, proteins and essential oils [10]. Several approaches have been tried to improve the bioavailability of curcumin. These approaches include the use of adjuvants such as piperine, the use of liposomal curcumin, curcumin nanoparticles, the use of curcumin phospholipid complex, and the use of structural analogs of curcumin $[9,10]$. It has been reported that heat treatment improves the water solubility of curcumin [10]. Piperine is an inhibitor of curcumin glucuronidation in the liver [21]. Shoba et al. reported that combined treatment with curcumin and piperine $(20 \mathrm{mg} / \mathrm{kg}$ ) produced higher serum concentration of curcumin from 0.25 to $1 \mathrm{~h}$ post-drug and increased the bioavailability by $2000 \%$ [22]. So, the clinical use of curcumin has been limited by its low solubility and poor oral bioavailability. Delivery by inhalation for the treatment of pulmonary disease likewise appears infeasible, because the organic solvents in which it must be dissolved are toxic to the lung. To overcome these drug-delivery obstacles, it has been developed a new formulation of curcumin in which it is complexed with hydroxypropyl ether derivatives of cyclodextrin (CDC). This formulation of curcumin is stable, highly water-soluble, bioavailable, and easily produced. This permits administration by either the intravenous or inhalation routes at doses that have been shown to elicit anti-inflammatory effects. CDC is rapidly cleared from the circulation after intravenous administration. The oral bioavailability of curcumin is low because of metabolism in the intestine, which would presumably affect oral $C D C$ as well. These limitations make inhalation a viable route of administration for $\mathrm{CDC}$, particularly for the targeted treatment of inflammatory lung disease [25]. In human trials, only a minor side effect of curcumin, namely diarrhea [26], has been reported. Curcumin is considered safe and well tolerated [10]. Even though curcumin exhibits a wide variety of pharmacological activities and has been found to be quite safe in humans, there are some reports concerning its toxicity. It has been reported that curcumin exhibit both antioxidant and pro-oxidant activities. It is possible that these opposing activities of curcumin are regulated by its concentration, so the effect of curcumin may switch from antioxidant to pro-oxidant. Nevertheless no long-term studies have been conducted to show curcumin toxic or adverse effects so far. Such studies are necessary in human subjects to determine the safety of curcumin in a long period of time [21].

\section{The hypothesis}

The problem of IPAH treatment is that available drugs are few, expensive, even more in a combination therapy, and they have various side effects. We propose curcumin as possible therapeutic solution for IPAH. It inhibits main pathways underlying this disease. Curcumin exhibits suppression on the NF- $\kappa \mathrm{B}$ pathway determining, through this way, also a control on inflammatory cytokines IL-1 $\beta$, IL- 6 and IL-8. Through the same way it controls COX2 production and COX2 induction. Curcumin inhibits TNF- $\alpha$, another element that takes part to the inflammatory process. It modulates inducible nitric oxide synthase (iNOS), 5-LOX, and phospholipases A2 (PLA2). So, on one hand, there is a control and inhibition of the inflammatory process and consequently of the inflammatory infiltrate. On the other hand, there is a control on the arterial remodeling. NF- $\kappa \mathrm{B}$ inhibition determines a suppression of the expression of cell proliferation genes. The modulation of inducible nitric oxide synthase (iNOS) controls the action of cGMP, giving vasodilation and anti-proliferation. The modulation of COX2, 5-LOX and PLA2 explicates a control on the arachidonic acid pathway and consequently on prostacyclin which gives vasodilation and anti-proliferation as well. Curcumin is a low cost therapeutic solution, well tolerated. The existing trials did not show side effects, except for diarrhea. Even though there are no long term studies on humans. Its main limits are poor bioavailability, rapid metabolism, low gastrointestinal absorption, rapid systemic elimination and low solubility in water. The new formulation of curcumin, complexed with hydroxypropyl ether derivatives of cyclodextrin is stable, highly water-soluble, bioavailable, and easily produced. So it seems to bridge the limits, permitting an administration by either the intravenous or inhalation routes.

\section{Possible clinical investigation}

So far it is known oral curcumin pharmacokinetics. A first step is to evaluate oral curcumin specific pharmacodynamics for IPAH. It is possible to do it confronting curcumin with a placebo. So, if oral curcumin shows good efficacy it should be tested in comparison with the standard therapy. In a second step it is possible to proceed from two results that we could obtain from the previous step. If oral curcumin does not show a major efficacy on the standard therapy, other types of formulation could be tested in comparison with it. These formulations could be then administered intravenously or via inhalation. Otherwise, if oral curcumin shows a major efficacy, pharmacokinetics should be increased and it is necessary to ameliorate patient compliance to therapy. In both cases new curcumin formulations are required possibly starting from $\mathrm{CDC}$ which has been already developed. Either the intravenous or the inhalatory formulation has to be studied in pharmacokinetics. Subsequently they have to be studied in their pharmacodynamics toward IPAH, firstly in comparison with a placebo. If good efficacy is achieved, they have to be tested secondly in comparison with the standard therapy.

\section{Conclusions}

Curcumin has been used for thousands of years in Ayurvedic medicine to treat various diseases. In the last decades it has been 
studied its mechanisms of activity and its pharmacological properties. It has been considered a potent anti-inflammatory agent useful for inflammatory diseases. Along the years its poor bioavailability and rapid metabolism have limited its therapeutic use. For this reason some studies have developed new formulations of curcumin complexed with other molecules. Curcumin affects numerous molecular targets and pathways, so in our opinion it could be used as therapeutic agent in IPAH. Clearly some studies are required to evaluate its efficacy on this disease. We propose herein an idea of how a clinical trial could be designed.

\section{Conflict of interest}

Nothing to disclose.

\section{References}

[1] McLaughlin VV, Archer SL, Badesch DB, et al. ACCF/AHA 2009 expert consensus document on pulmonary hypertension: a report of the American College of cardiology foundation task force on expert consensus documents and the American Heart Association: developed in collaboration with the American College of Chest Physicians, American Thoracic Society, Inc., and the Pulmonary Hypertension Association. Circulation 2009;119(16):2250-94.

[2] Price LC, Wort SJ, Perros F, et al. Inflammation in pulmonary arterial hypertension. Chest 2012;141(1):210-21.

[3] Schermuly RT, Ghofrani HA, Wilkins MR, Grimminger F. Mechanisms of disease: pulmonary arterial hypertension. Nat Rev Cardiol 2011;8(8):443-55.

[4] Rabinovitch M. Molecular pathogenesis of pulmonary arterial hypertension. J Clin Invest 2008;118(7):2372-9.

[5] McLaughlin VV, Davis M, Cornwell W. Pulmonary arterial hypertension. Curr Probl Cardiol 2011;36(12):461-517.

[6] Simonneau G, Robbins IM, Beghetti M, et al. Updated clinical classification of pulmonary hypertension. J Am Coll Cardiol 2009;54(Suppl. 1):S43-54.

[7] Ulrich S, Fischler $M$, Speich R. Update on therapies for pulmonary hypertension. Swiss Med Wkly 2007;137(5-6):73-82.

[8] Rosenkranz S. Pulmonary hypertension: current diagnosis and treatment. Clin Res Cardiol 2007;96(8):527-41.

[9] Zhou H, Beevers CS, Huang S. The targets of curcumin. Curr Drug Targets 2011;12(3):332-47.

[10] Epstein J, Sanderson IR, Macdonald TT. Curcumin as a therapeutic agent: the evidence from in vitro, animal and human studies. $\mathrm{Br} J$ Nutr 2010;103(11):1545-57.
[11] Bharti AC, Donato N, Aggarwal BB. Curcumin (diferuloylmethane) inhibits constitutive and IL-6-inducible STAT3 phosphorylation in human multiple myeloma cells. J Immunol 2003;171(7):3863-71.

[12] Strimpakos AS, Sharma RA. Curcumin: preventive and therapeutic properties in laboratory studies and clinical trials. Antioxid Redox Signal 2008;10(3):511-45.

[13] Baeuerle PA, Henkel T. Function and activation of NF-kappa B in the immune system. Annu Rev Immunol 1994;12:141-79.

[14] Tak PP, Firestein GS. NF-kappaB: a key role in inflammatory diseases. J Clin Invest 2001;107(1):7-11.

[15] Munford RS, Pugin J. Normal responses to injury prevent systemic inflammation and can be immunosuppressive. Am J Respir Crit Care Med 2001;163(2):316-21.

[16] Abe Y, Hashimoto S, Horie T. Curcumin inhibition of inflammatory cytokine production by human peripheral blood monocytes and alveolar macrophages. Pharmacol Res 1999;39(1):41-7.

[17] Srimal RC, Dhawan BN. Pharmacology of diferuloyl methane (curcumin), a non-steroidal anti-inflammatory agent. J Pharm Pharmaco 1973:25(6):447-52.

[18] Kumar A, Dhawan S, Hardegen NJ, Aggarwal BB. Curcumin (diferuloylmethane) inhibition of tumor necrosis factor (TNF)-mediated adhesion of monocytes to endothelial cells by suppression of cell surface expression of adhesion molecules and of nuclear factor-kappaB activation. Biochem Pharmacol 1998;55(6):775-83.

[19] Lao CD, Ruffin 4th MT, Normolle D, et al. Dose escalation of a curcuminoid formulation. BMC Complement Altern Med 2006;17(6):10.

[20] Cheng AL, Hsu CH, Lin JK, et al. Phase I clinical trial of curcumin, a chemopreventive agent, in patients with high-risk or pre-malignant lesions. Anticancer Res 2001;21(4B):2895-900.

[21] Aggarwal BB, Harikumar KB. Potential therapeutic effects of curcumin, the anti-inflammatory agent, against neurodegenerative, cardiovascular pulmonary, metabolic, autoimmune and neoplastic diseases. Int J Biochem Cell Biol 2009;41(1):40-59.

[22] Shoba G, Joy D, Joseph T, Majeed M, Rajendran R, Srinivas PS. Influence of piperine on the pharmacokinetics of curcumin in animals and human volunteers. Planta Med 1998;64(4):353-6.

[23] Sharma RA, Gescher AJ, Steward WP. Curcumin: the story so far. Eur J Cancer 2005;41(13):1955-68

[24] Vareed SK, Kakarala M, Ruffin MT, et al. Pharmacokinetics of curcumin conjugate metabolites in healthy human subjects. Cancer Epidemiol Biomarkers Prev 2008:17(6):1411-7.

[25] Suresh MV, Wagner MC, Rosania GR, et al. Pulmonary administration of a water-soluble curcumin complex reduces severity of acute lung injury. Am J Respir Cell Mol Biol 2012:47(3):280-7.

[26] Sharma RA, Euden SA, Platton SL, et al. Phase I clinical trial of oral curcumin: biomarkers of systemic activity and compliance. Clin Cancer Res 2004;10(20):6847-54. 